\title{
APLICAÇÃO DA LEI DE BRADFORD A PESQUISAS RELACIONADAS A OPEN GOVERNMENT
}

\author{
Márcio José Sembay* \\ Centro Universitário UNIFACVEST \\ Adilson Luiz Pinto ${ }^{* *}$ \\ Universidade Federal de Santa Catarina \\ Douglas Dyllon Jeronimo de Macedo ${ }^{* * *}$ \\ Universidade Federal de Santa Catarina \\ José Antonio Moreiro González ${ }^{* * *}$ \\ Departamento de Biblioteconomia e Documentação. Universidad Carlos III de Madrid.
}

\begin{abstract}
Resumo: Objetivou-se analisar a produção cientifica sobre o termo Open Government na base de dados Web of Science no período de 2010 a 2016, usando como filtro de busca os títulos dos artigos em todas as linguagens, considerando a distribuição das produções intelectuais internacionais. A pesquisa recuperou os periódicos mais relevantes da área de ciências sociais aplicadas, observando seu impacto para esta área de conhecimento. A pesquisa tem caráter quantitativo, pois analisou-se a produção cientifica com acesso online da base de dados Web of Science abrangendo um total de 3.165 registros. Aplicou-se uma das principais leis da bibliometria, a lei de Bradford para essa análise. Utilizou-se uma ferramenta de planilha para análise da mineração, tabulação e tratamento dos dados. O trabalho revela a não linearidade nas publicações ao longo dos anos registrando um aumento significativo na produção em um contexto internacional. Conclui-se que o termo Open Government tem qualidade em produções científicas conforme o núcleo da lei de Bradford demonstrada nesse estudo, porém, o termo ainda está ganhando espaço em publicações cientificas na atualidade em uma ciência em transformação.
\end{abstract}

Palavras-chave: Bibliometria; lei de Bradford; dispersão da literatura cientifica; open government; periódicos científicos; Web of Science.

Resumen: Se ha buscado analizar la producción científica sobre el término Open Government en la base de datos Web of Science durante el período de 2010 a 2016, usando como filtro de búsqueda los títulos de los artículos en todas las lenguas y considerando la dispersión de la producción intelectual internacional. La investigación seleccionó las revistas más relevantes del área de ciencias sociales aplicadas, observando el impacto de esas revistas en esta área de conocimiento. La investigación es de carácter cuantitativo, pues se analizó la producción científica a través del acceso en línea a la base de datos Web of Science que alcanzó hasta 3.165 registros totales. Para hacer el análisis de la dispersión de la literatura afectada se aplicó la ley bibliométrica de Bradford. Se empleó como herramienta de hoja de cálculo para analizar los datos obtenidos en la minería, así como para tabularlos y tratarlos. El trabajo revela que, a lo largo de los años, las publicaciones muestran una aparición no lineal, registrándose un aumento significativo en la producción relativa al contexto internacional. De acuerdo con el núcleo de la ley de Bradford, se demuestra que el término Open Government muestra calidad en las publicaciones científicas en las que se difunde, pero que aún se encuentra en una fase de lucha por su espacio en las publicaciones científicas dentro de una ciencia actual en transformación.

Palabras clave: Bibliometría; Ley de Bradford; dispersión de la literatura científica; open government; revistas científicas; Web of Science

Título: APLICACIÓN DE LA LEY DE BRADFORD A LA INVESTIGACIÓN SOBRE OPEN GOVERNMENT.

\begin{abstract}
The objective of this study was to analyze the scientific production of the term Open Government in the Web of Science database from 2010 to 2016, using as search filter the titles of articles in all languages, considering the distribution of international intellectual productions. The research retrieved the most relevant journals in the area of applied social sciences, observing the impact of these journals to this area of knowledge. The research has a quantitative character, as it was analyzed the scientific production with online access of the Web of Science database covering a total of 3,165 documents. One of the main laws of bibliometrics was applied, Bradford's law for this analysis. If you used a spreadsheet tool for mining analysis, tabulation and data processing. The work reveals non-linearity in publications over the years recording a significant increase in production in an international context. It is concluded that the term Open Government has quality in scientific productions according to the core of the law
\end{abstract}

\footnotetext{
*m_sembay@yahoo.com.br

**adilson.pinto@ufsc.br

*** douglas.macedo@ufsc.br

**** jamore@bib.uc3m.es
}

Recibido: 05-04-2018; 2a versión: 22-05-2019; 3ª versión: 24-09-2019; aceptado: 26-09-2019.

SEMBAY, M.J.; PINTO, A.L.; MACEDO, D.D.J. y MOREIRO GONZÁLEZ, J.A. Aplicación de la Ley de Bradford a la investigación sobre Open Government. Anales de Documentación, 2020, vol. 23, $\mathrm{n}^{\circ} 1$. Disponible en: http://dx.doi.org/10.6018/analesdoc.326771. 
of Bradford demonstrated in that study, however, the term is still gaining space in scientific publications nowadays in a society in transformation.

Keywords: Bibliometric; Bradford's law; dispersion of scientific literature; open government; scientific journals; Web of Science.

Title: APPLICATION OF THE BRADFORD LAW TO RESEARCH RELATED TO OPEN GOVERNMENT.

Copyright: (C) 2020 Servicio de Publicaciones de la Universidad de Murcia (Spain). Este es un artículo de acceso abierto distribuido bajo los términos de la licencia Creative Commons Reconocimiento 4.0 Internacional (CC BY 4.0).

\section{INTRODUÇÃ̃O}

O termo bibliometria que se utiliza nos estudos métricos da informação foi proposto por Pritchard (1969) e pode ser definido como a aplicação de métodos estatísticos e matemáticos na análise de obras literárias.

No campo das ciências sociais aplicadas, os estudos bibliométricos se concentram em examinar a produção de artigos em um determinado campo do saber, mapear as comunidades acadêmicas e identificar as redes de pesquisadores e suas motivações, desse modo, tais objetivos são vistos de modo tangível por meio da criação de indicadores que buscam sumarizar as instituições e os autores mais prolíferos, os acadêmicos mais citados e as redes de coautorias (Okubo, 1997).

Em relação as leis que regem os estudos bibliométricos podem se aplicar três leis empíricas sobre o comportamento da literatura: o método de medição da produtividade de cientistas de Lotka, a lei de dispersão do conhecimento científico de Bradford e o modelo de distribuição e frequência de palavras num texto de Zipf (TagueSutcliffe, 1992). A lei de Bradford ou Lei de Dispersão permite, com a mensuração da produtividade dos periódicos, indicar o núcleo e as áreas dispersas a respeito de um assunto no mesmo conjunto de periódicos. Auxilia no desenvolvimento de políticas de aquisição e descarte de periódicos sobre determinados assuntos (Tague-Sutcliffe, 1992).

Para abordar o pressuposto de estudo se aplicou a lei de Bradford na busca por periódicos na base de dados Web of Science $(W o S)$ de 2010 a 2016 em relação ao termo Open Government. A escolha desta base de dados se dá pela sua cobertura de conteúdos relacionados desde 1945, e também por uma dinâmica fácil e ágil de disposição dos dados para extração, filtragem, tratamento e aplicação de métricas.

A problemática analisada é a relação das distribuições estatísticas e gráficas dos resultados encontrados na pesquisa gerados pelas regras da lei de Bradford, relacionando esses resultados ao objetivo de analisar a literatura de Open Government, a fim de verificar a existência de um núcleo representativo de periódicos através da lei aqui aplicada.

Nesse sentido cabe ressaltar, segundo Ferrer, Peset e Benavent (2011), que Open Government é a forma de comunicação aberta e permanente entre os governos e seus cidadãos, baseada na transparência, gestão e colaboração, podendo ser formada uma parceria visando melhorias para todos. Essa relação faz uso intenso de recursos tecnológicos, tais como redes sociais. Uma das principais ferramentas para que iniciativas de Open Government efetivamente atinjam seus objetivos é através de dados abertos.

Portanto, uma breve revisão de literatura sobre os conceitos dos assuntos mais pertinentes e que nortearam esse estudo serão apresentados nas próximas seções, assim como a metodologia que possibilitou a busca e mensuração dos dados e logo após os resultados e comentários e as conclusões sobre a pesquisa em questão.

\subsection{Estudos métricos a partir da lei de Bradford}

A lei de Bradford, ou lei da dispersão, incide sobre um conjunto de publicações (periódicos) para determinar o núcleo dos mais produtivos em uma determinada temática. Sua representação é realizada por zonas, e sua distribuição é apresentada em três zonas, sendo todas representadas por $33 \%$ das publicações (Bradford, 1934).

A ideologia deste tipo de análise surgiu a partir de uma necessidade encontrada nas informações de periódicos nas áreas de ciências médicas nos Estados Unidos da América, no qual visava identificar a extensão das revistas científicas em um assunto específico. Os dados coletados mostraram a existência de um pequeno núcleo de periódicos que abordavam o assunto de maneira mais extensiva, e uma vasta região periférica dividida em duas zonas de um número elevado de títulos de revistas com pouca representação na temática estudada. 
Em síntese, o estudo determinou que para a temática de estudo existem poucas revistas especializadas no assunto específico, e ao mesmo tempo um universo grande de revistas que tratam do tema esporadicamente. Então, o foco de informação pode ser minimizado por poucos títulos que detém um alto grau de representatividade.

Brookes (1969) explica que se periódicos científicos forem ordenados de maneira decrescente de produtividade de artigos, sobre determinado assunto, poderão ser divididos em um núcleo de periódicos especificamente ligados ao assunto e, em vários grupos ou zonas, contendo o mesmo número de artigos que o núcleo. O número de periódicos (n), no núcleo e zonas subsequentes, variará na proporção 1:n:n². A Figura 1 apresenta a fórmula da lei de Bradford e estão representadas em três zonas de Bradford.

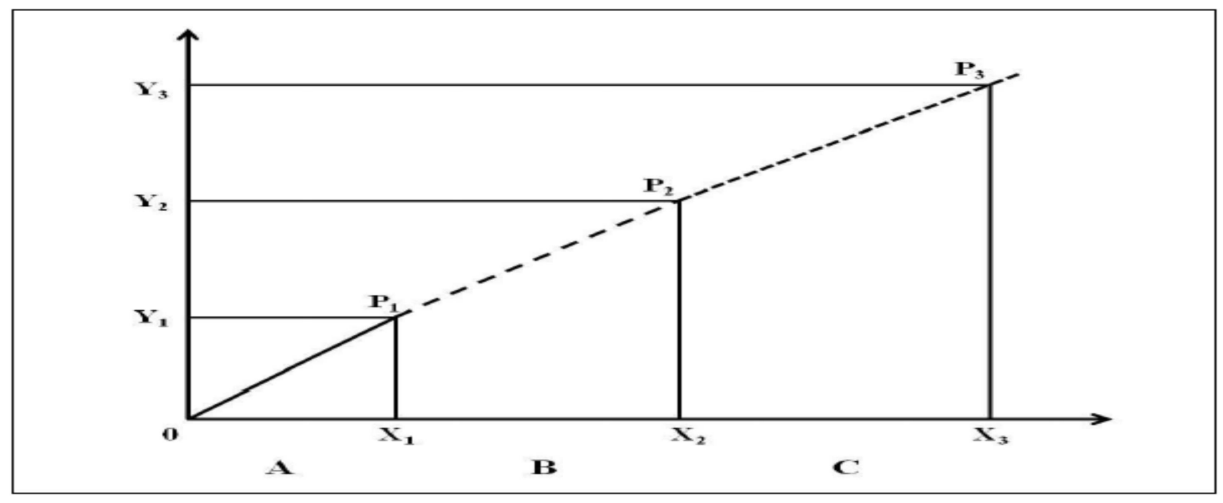

Figura 1. Lei de Bradford. Fonte: Pinheiro, 1983.

Explica-se que a Zona $A$ corresponde à concentração; a Zona $B$ é correspondente à produtividade média e pode ser apresentada como a informação interessante, que pode ser estudada em um segundo momento; e a Zona $C$ compreende os periódicos de baixa produtividade (PINHEIRO, 1983), que podemos chamar também de ruído.

Utilizando a lei de Bradford, e sua forma de aplicabilidades, nossa proposta visa demonstrar como o termo Open Government se encontra na modelagem do núcleo e zonas de frequência pesquisadas na base de dados $W o S$.

\subsection{Open government, o termo e o conceito}

O termo Open Government não é novo, e ao longo da história, esteve associado ao direito de acesso à informação pública, o qual foi primeiramente regulado na Suécia, ainda em 1766. Dois séculos mais tarde, a Lei de Liberdade de Informação (Freedom of Information Act, FOIA) foi promulgada nos Estados Unidos, em 1966. Em documentos que deram origem à FOIA, já era possível encontrar o termo Open Government, em alusão ao direito de saber e ao direito à informação (Peled, 2013; Yu e Robinson, 2012; Güemes e Ramírez-Alujas, 2012).

Helbig et al. (2012) apontam que, através de projetos de Open Government utilizando as Tecnologias de Informação e Comunicação, os governos podem obter diversos benefícios. Para o que empregam a expressão "debaixo do guarda-chuva de Open Government". Resumem esses benefícios em dois grandes focos: (i) internamente os governos estão procurando melhorar sua produtividade, através da utilização dos cidadãos para colaborarem nos trabalhos ou proporcionando aos seus servidores novas possibilidades para orientar os cidadãos em melhorar seus comportamentos e; (ii) externamente, os governos estão procurando maximizar o potencial dos cidadãos para fiscalizar o governo, proporcionando aos cidadãos uma maior escolha (pela maior divulgação de informações) ou visando estimular o desenvolvimento econômico através do uso e reuso de informações dos governos.

É importante ressaltar que Open Government, dados abertos e dados governamentais abertos têm significados diferentes, ou seja, pode-se resumir que Open Government é disponibilização de informações em qualquer formato por parte dos governos e outras ações que visem promover maior transparência. Dados abertos é a disponibilização de informações, em alguns formatos (pré-estabelecidos), por governos, organizações privadas, com ou sem fins lucrativos, ou por outros atores de uma sociedade. Dados governamentais abertos é a disponibilização de dados por parte dos governos, nos mesmos formatos estabelecidos para dados abertos (Luna-Reyes e Gil-García, 2013).

Na figura 2 podemos visualizar a conceituação anteriormente dita de forma mais clara. Nela se observa que os dados governamentais abertos se tornam mediadores entre o Open Government e os dados abertos. 


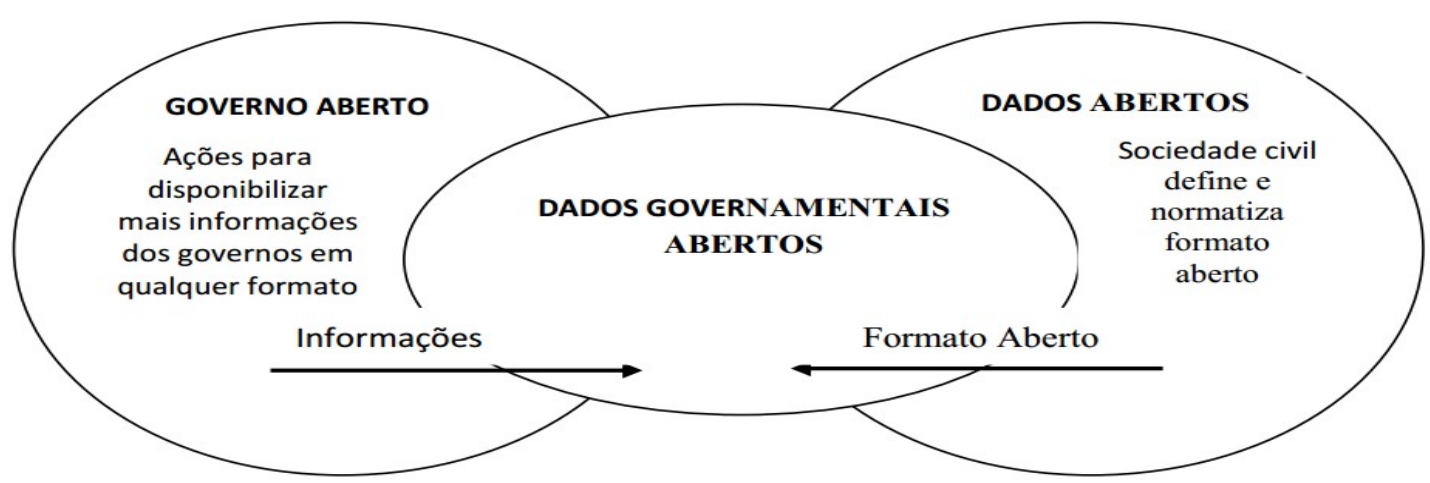

Figura 2. Open Government, dados abertos e dados governamentais abertos. Fonte: Adaptado de Luna-Reyes e Gil-Garcia (2013).

\section{METODOLOGIA}

Seguindo a identificação das zonas de publicação na temática de Open Government, este estudo se baseia na proposta de Pinheiro (1983) e tem como aplicação o método de ordenamento informacional de Brookes (1969), caracterizado pela hierarquia decrescente de aparições da temática de estudo, como $1: \mathrm{n}: \mathrm{n}^{2}: \mathrm{n}^{3}$.

Para a aplicação 'n' foi denominado como multiplicador de Bradford e tem o objetivo de ser o núcleo de relevância (as revistas mais incisivas na temática - especializadas); " $\mathrm{n}^{2}$ " é a Zona 1 (não possuem uma frequência elevada de artigos sobre a temática, porém podem ser consultadas em uma possível segunda etapa), e " $n$ " é a Zona 2 (possui muito ruído, afinal apresentam uma frequência baixa para a temática de estudo).

\section{PERIÓDICOS}

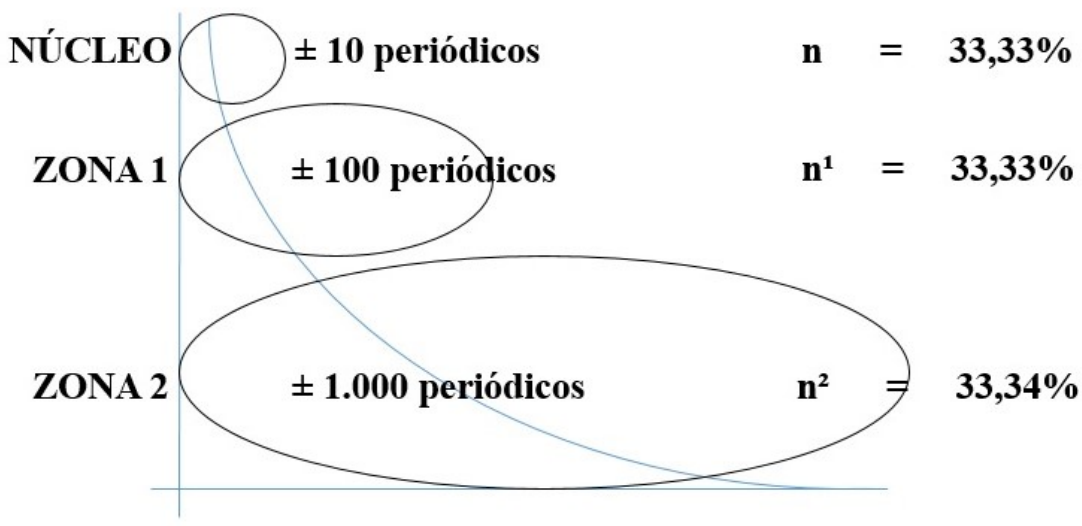

Figura 3. Modelo de tabela da aplicação da lei de Bradford. Fonte: Baseado em Pinheiro (1983).

Para a recuperação dos documentos pertinentes à temática trabalhada foi realizada uma pesquisa avançada (por Topic) na base de dados Web of Science (recuperação por título do artigo, resumo e palavras-chave) como fonte para levantamento dos dados (TS=Open Government); e posteriormente foi realizado um refinamento por tipo de documento (Document Types $=$ Article). $\mathrm{O}$ termo buscado somente em inglês se dá pelo fato que a base de dados em questão indexa em inglês todos os seus conteúdos, ficando normalizada a sua busca e recuperação.

O estudo se baseou em uma análise temporal que transcorreu de 2010 a 2016 e utilizou o refinamento pelos índices de citação Science Citation Index Expanded (SCI-EXPANDED), Social Sciences Citation Index (SSCI) e Arts \& Humanities Citation Index (A\&HCI).

Com os refinamentos iniciais, a pesquisa recuperou um total de 3,165 documentos/artigos relacionados a terminologia de busca. 
De posse dos documentos, criamos uma base tabular (em forma de planilhas), no qual foi possível realizar as análises e a aplicação da lei de Bradford para os títulos dos periódicos em que estes documentos/artigos foram publicados (tabela I).

Logo em seguida, geramos algumas dinâmicas no sistema tabular para identificar o núcleo da temática e as demais zonas (tabela 2), representados por:

$$
\begin{aligned}
& A=\text { frequência de periódicos; } \\
& B=\text { número de artigos por periódico; } \\
& C=\text { 'A' acumulado; } \\
& D=\text { 'B' acumulado; } \\
& \text { Ln }(C)=\text { logaritmo natural de } C, e ; \\
& A * B=\text { subtotal de artigos. }
\end{aligned}
$$

Foram utilizadas fórmulas matemáticas para a aplicação da lei de Bradford, visando saber seu cálculo multiplicador (Egghe, 1986), (Egghe, 1990) e (Egghe e Rousseau, 1990):

Onde:

$$
k=\left(1,781 \times Y_{m}\right)^{\frac{1}{P}}
$$

$\mathrm{Y}_{\mathrm{m}}=$ A produtividade máxima $\left(1^{\mathrm{o}}\right.$ valor da coluna $\left.\mathrm{D}\right)$

$\mathrm{P}=$ Número de zonas.

O número de zonas foi obtido a partir de 3 e foi aumentando conforme foi necessário dentro da análise dos dados recuperados.

Para realizar o cálculo do número de periódicos no núcleo, T é representado como o número Total de periódicos, sendo o último valor da coluna $\mathrm{C}$.

$$
r_{o}=\frac{T \times(k-1)}{\left(k^{P}-1\right)}
$$

Para o cálculo do número de periódicos nas demais zonas, se utilizou a proporção $1: \mathrm{k}: \mathrm{k}^{2}: \mathrm{k}^{3}: .$. , sendo observado como:

$$
\begin{aligned}
& \text { Núcleo }=r_{\mathrm{o}} \times 1 \\
& \text { Zona } 1=\mathrm{r}_{\mathrm{o}} \times \mathrm{k} \\
& \text { Zona } 2=\mathrm{r}_{\mathrm{o}} \times \mathrm{k}^{2} \\
& \text { Zona } 3=\mathrm{r}_{\mathrm{o}} \times \mathrm{k}^{3}
\end{aligned}
$$

Para o cálculo da equação da curva de Bradford (gráfico 1), foi utilizado $R(r)=a \times \ln (1+b \times r)$, conforme Leimkuhler (1967).

Para sua aplicabilidade temos R(r) que é o número de artigos por periódicos acumulados, cujo ranking é r, ou seja, $R(r)$ são os valores da coluna $D$, que podem ser melhor visualizados na tabela 2 .

\section{RESULTADOS E DISCUSSÃO}

Para o desenvolvimento de um estudo que visa saber como estão as informações abertas sobre governos, foi relevante fazer um diagnóstico inicial para saber qual o período mais crítico de informações sobre a temática. Em uma análise inicial na $W o S$ conseguimos identificar que o período a partir de 2010 foi o mais expressivo, saltando de poucas aparições (até 2009) do termo na referida base de dados para mais de três centenas em um único ano. Isso representa a importância da discussão mundial sobre deixar aberta a informação sobre como é gerada e monitorada a informação pública/governamental. Acreditá-se que tenha dois motivos por trás deste período, (i) quando os países começam a validar a maioria das leis de acesso a informação, mesmo que alguns tenham este instrumento legal a mais tempo, e; (ii) tem a ver com o comunicado, em 2006, do Comitê de Direitos Humanos das Nações Unidas em conjunto com a Corte Europeia de Direitos Humanos e a Corte Interamericana de Direitos Humanos salientando o direito do cidadão a informação púbica, que leva um tempo para ser digerida pela sociedade científica e política. 
Visando atender esta demanda, que foi constatada nas buscas, a pesquisa seguiu os passos metodológicos, no qual foi possível identificar o núcleo de periódicos involucrados em sanar as inquietações sobre o tema, categorizados por 187 títulos, no qual representamos na tabela 1 somente os periódicos mais representativos com um índice igual ou superior a $\mathrm{n}=11$. Dentro da análise existe a possibilidade da dispersão na literatura em relação aos assuntos específicos, visto que o fato do tema pesquisado ser tratado por um grande número de periódicos, aumenta o risco de haver um número representativo de periódicos não indexados.

\begin{tabular}{lll}
\hline TÍTULOS DA FONTE & REGISTROS & \% de 3165 \\
\hline GOVERNMENT INFORMATION QUARTERLY & 70 & 2.212 \\
ECONOMIC MODELLING & 24 & 0.758 \\
PLOS ONE & 24 & 0.758 \\
ENERGY POLICY & 18 & 0.569 \\
SOCIAL SCIENCE MEDICINE & 17 & 0.537 \\
LAND USE POLICY & 16 & 0.506 \\
MARINE POLICY & 16 & 0.506 \\
BMC PUBLIC HEALTH & 13 & 0.411 \\
BMC HEALTH SERVICES RESEARCH & 12 & 0.379 \\
CITIES & 12 & 0.379 \\
JOURNAL OF INTERNATIONAL MONEY AND FINANCE & 12 & 0.379 \\
PUBLIC ADMINISTRATION & 12 & 0.379 \\
SUSTAINABILITY & 12 & 0.379 \\
HEALTH POLICY & 11 & 0.348 \\
JOURNAL OF CLEANER PRODUCTION & 11 & 0.348 \\
SOCIAL SCIENCE COMPUTER REVIEW & 11 & 0.348 \\
.. & $\cdots$ & \\
\hline
\end{tabular}

Tabela I. Principais periódicos sobre o termo Open Government. Fonte: Dados da pesquisa.

Os dados apresentados na tabela 1, mostram o periódico Government Information Quarterly como o mais destacado. Buscamos saber um pouco mais sobre este periódico internacional que trabalha sobre Gestão, Políticas e Práticas de Tecnologia da Informação, com um fator de impacto de 4.090, conforme coletado em seu site em janeiro de $2018^{1}$. Esse periódico contabilizou $n=101$ registros de pesquisas no estudo, representando $2,2 \%$ do total de pesquisas relacionadas à temática estudada.

Também observou a relação do tema central de pesquisa com periódicos das mais variadas áreas do conhecimento, e inclusive com aporte multidisciplinar, devido a extensão que Open Government pode ser explorado, como são os periódicos de destaque, como na área de Economia (Economic Modelling, CITIES, Land Use Policy e Marine Policy), Saúde (Health Policy, BMC Public Health e BMC Health Services Research), Administração/Finanças (Journal of International Money and Finance e Public Administration), Ciências Sociais (Social Science Computer Review e Social Science Medicine), Meio Ambiente (Journal of Cleaner Production e Sustainability), Energia (Energy Policy) e Multidisciplinar (Plos ONE).

$\mathrm{N}$ tabela 2 a seguir, contém os dados da tabela 1, transformada em uma planilha dinâmica, sumarizando os dados da tabela 1, e agrupando os dados para a tabela 2 em zonas com um número aproximado de artigos.

\begin{tabular}{llllll}
\hline $\mathrm{A}$ & $\mathrm{B}$ & $\mathrm{C}$ & $\mathrm{D}$ & $\mathrm{Ln}(\mathrm{C})$ & $\mathrm{A} * \mathrm{~B}$ \\
\hline 1 & 70 & 1 & 70 & 0 & 70
\end{tabular}




\begin{tabular}{llllllll} 
& 2 & 24 & 3 & 94 & 0,477121 & 48 & \\
& 1 & 18 & 4 & 112 & 0,60206 & 18 & \\
& 1 & 17 & 5 & 129 & 0,69897 & 17 & \\
& 2 & 16 & 7 & 145 & 0,845098 & 32 & \\
& 1 & 13 & 8 & 158 & 0,90309 & 13 & \\
& 5 & 12 & 13 & 170 & 1,113943 & 60 & \\
& 3 & 11 & 16 & 181 & 1,20412 & 33 & \\
& 2 & 10 & 18 & 191 & 1,255273 & 20 & \\
187 & 9 & 9 & 27 & 200 & 1,431364 & 81 & \\
Núcleo & 8 & 8 & 35 & 208 & 1,544068 & 64 & 1164 \\
& 12 & 7 & 47 & 215 & 1,672098 & 84 & \\
& 16 & 6 & 63 & 221 & 1,799341 & 96 & \\
& 32 & 5 & 95 & 226 & 1,977724 & 160 & \\
\hline 453 & 92 & 4 & 187 & 230 & 2,271842 & 368 & \\
Zona 1 & 329 & 3 & 316 & 233 & 2,499687 & 387 & \multirow{2}{*}{1035} \\
\hline 966 & 2 & 640 & 235 & 2,80618 & 648 & \\
Zona 2 & 966 & 1 & 1615 & 236 & 3,208173 & 966 & \multirow{2}{*}{966} \\
\hline Tabela II. Dados sumarizados da tabela 1 com as divisões de cada zona. Fonte: Dados da pesquisa.
\end{tabular}

Pode-se observar na tabela 2, que os primeiros 187 periódicos são dedicados ao termo Open Government, ou seja, o núcleo dessa tabela no estudo em questão. Assim, pode-se afirmar que $30 \%$ do total, conforme a multiplicação da frequência de periódicos com o número de artigos por periódico $\left(\mathrm{A}^{*} \mathrm{~B}=\right.$ subtotal de artigo) se encontrou mais acentuada.

Ainda na tabela 2, foram realizadas as divisões das zonas, onde cada zona teve aproximadamente a mesma porcentagem de artigos, ou seja, foram divididos em três zonas: núcleo, zona 1 e zona 2. Para a conferência da lei de Bradford, onde:

- O núcleo teve 187 periódicos, totalizando $36,77 \%$ do total de artigos;

- Zona 1 teve 453 periódicos, totalizando $32,70 \%$ do total de artigos;

- Zona 2 teve 966 periódicos, totalizando $30,53 \%$ do total de artigos.

Em relação à representação das zonas, há uma dispersão considerável de uma zona para a outra, sendo que, um ponto relevante a ser ressaltado na tabela 2 em relação ao núcleo a partir da aplicação da lei de Bradford, é que nesta zona existe perfeitamente um importante aspecto relativo ao comportamento acadêmico científico, ou seja, um núcleo de periódicos que supostamente indicam serem os de maior qualidade ou relevância para o termo aqui pesquisado.

Nesse sentido, fazendo uma análise da tabela 2, agrupou-se os elementos dessa tabela em (A*B), no qual somou-se essa coluna retornando aproximadamente o total de 3.165 artigos, que dividido pela quantidade de zonas utilizadas, que neste caso foi 3 (três), gerou um total aproximadamente de 1055 artigos por zona.

Verificando as proporções, onde o cálculo é a (zona 2/zona 1) resultando em 2,15231 e a (zona 1/núcleo), resultando em 2,24246, temos o multiplicador de Bradford igual a 2.

Para o cálculo de $\mathrm{K}$ e R0 foram utilizadas as fórmulas especificadas na metodologia desse estudo, encontrando os seguintes valores: $\mathrm{K}=4,994661$ e $\mathrm{R} 0=52,19561$, sendo que, esses resultados mostram uma dispersão do ideal para o correto da lei de Bradford.

Nesse sentido, podemos considerar, que conforme o estudo do termo Open Government for sendo mais conhecido e aplicado em pesquisas, além de aumentar o volume de artigos no núcleo, também ocorrerá uma maior dispersão de publicações em periódicos que ainda não estão sendo contempladas no estudo aqui em questão. Por isso, Brookes (1969) alerta sobre a necessidade de consideração de um período de tempo razoável. 
A tabela 3 demonstra o logaritmo natural da frequência acumulada de periódicos (Ln (C)) e o número acumulado de artigos por periódicos (D) registrados nessa tabela para posteriormente gerar a curva da lei de Bradford.

\begin{tabular}{|c|c|}
\hline $\operatorname{Ln}(\mathrm{C})$ & $\mathrm{D}$ \\
\hline 0 & 70 \\
\hline 0,477121 & 94 \\
\hline 0,60206 & 112 \\
\hline 0,69897 & 129 \\
\hline 0,845098 & 145 \\
\hline 0,90309 & 158 \\
\hline 1,113943 & 170 \\
\hline 1,20412 & 181 \\
\hline 1,255273 & 191 \\
\hline 1,431364 & 200 \\
\hline 1,544068 & 208 \\
\hline 1,672098 & 215 \\
\hline 1,799341 & 221 \\
\hline 1,977724 & 226 \\
\hline 2,271842 & 230 \\
\hline 2,499687 & 233 \\
\hline 2,80618 & 235 \\
\hline 3,208173 & 236 \\
\hline
\end{tabular}

Tabela III. Dados que geram a curva da lei de Bradford nesse estudo. Fonte: Dados da pesquisa.

A tabela 3 ressalta os dados numéricos que irão gerar a curva da lei de Bradford, na qual nesse estudo, buscou-se relatar as tendências para a temática em questão.

Por fim, o gráfico 1, a seguir, apresenta a curva da lei de Bradford gerada a partir da tabela 3, onde é ressaltada a produtividade de periódicos em relação ao termo Open Government, o número acumulado de artigos e o logaritmo do número acumulado de periódicos.

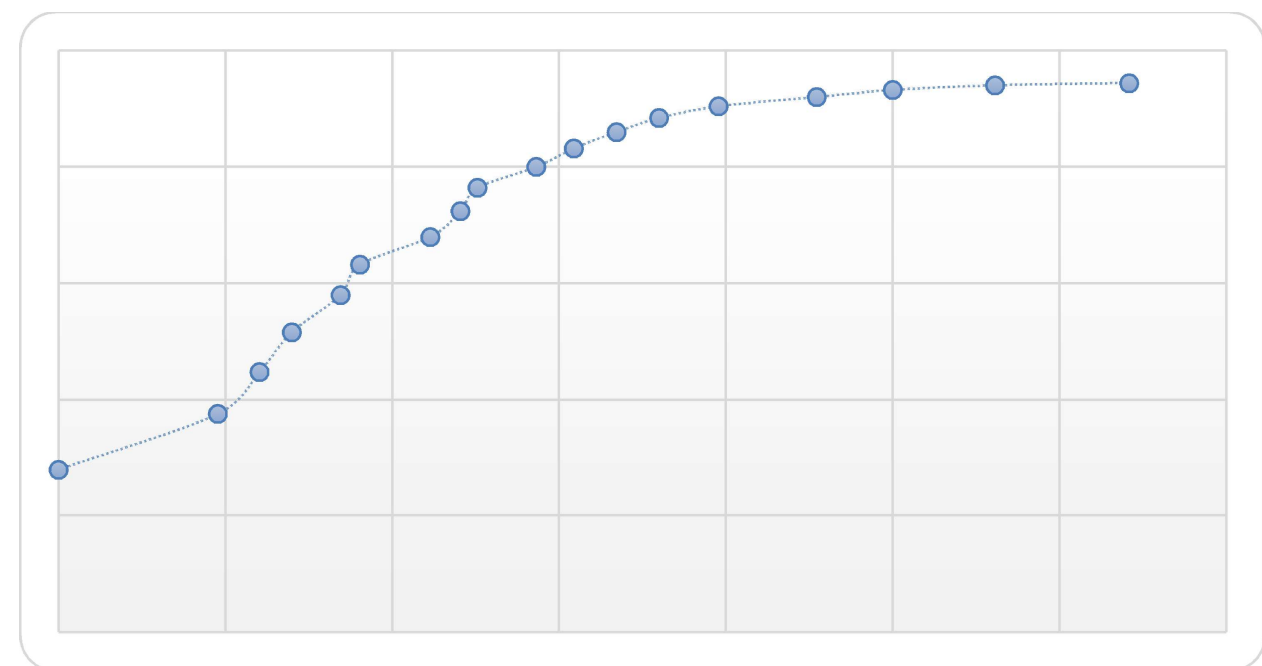

Gráfico 1. Produtividade de periódicos com o termo Open Government na Web of Science. Fonte: Dados da pesquisa.

Nesta análise, verificou-se a relação $1: \mathrm{n}: \mathrm{n}^{2}: \mathrm{n}^{3}$ :..., e foi testado com o núcleo +2 zonas. Observa-se também no gráfico 1, que a curva da lei de Bradford aplicado a esse estudo se encontra próximo aos padrões estabelecidos por 
essa lei, apesar de não chegar a traçar um "S" conforme as regras da lei aplicada. Conforme o multiplicador de Bradford encontrado nesse estudo, igual a 3, observou-se um crescimento considerável no gráfico 1 do número de artigos acumulados entre os intervalos de 50 a 250.

Ainda realizando uma inspeção visual do gráfico 1, observou-se que a tendência em publicações a respeito de Open Government é crescente, partindo do pressuposto que, o impacto das iniciativas de dados abertos atuais, estão resultando em um olhar para pesquisas sobre esse termo e a disseminação da política governamental de dados abertos e sua prática em todo o mundo.

Nesse sentido, o gráfico 1 ainda demonstra que a produtividade de periódicos sobre Open Government entre 2010 e 2016 está tendo uma proporção gradativa em publicações ao ponto que a própria sociedade está se inteirando sobre o assunto e dependendo do acesso aberto para geração e compartilhamento de informação e construção de conhecimento.

De fato, as hipóteses propostas por Brookes (1969), para a aplicação da lei de Bradford, podem justificar, em certa medida, o não ajustamento dos dados à distribuição ideal proposta por Bradford.

\section{CONSIDERAÇÕES FINAIS}

No presente estudo buscou trazer contribuições relacionadas à construção de indicadores bibliométricos que ressaltassem a pesquisadores atuantes em Open Government os principais periódicos onde a produção científica relacionada ao tema é publicada. Foi possível apresentar empiricamente e aplicar a lei de Bradford, que representa um importante parâmetro de análise de dispersão de publicações, mesmo que venha ser necessário ajustar algumas variáveis.

Conforme a aplicação da lei de Bradford nesse estudo, conclui-se que o termo Open Government evidencia a regra geral de que "poucos autores produzem muito e muitos autores produzem pouco" é verdadeira se consideradas as características da distribuição dos dados aqui pesquisados, apesar de alguns autores discutirem a validade dessa lei, os resultados encontrados nesse estudo tem a sua proposição confirmada.

Nesse sentido, é importante ressaltar que nesse estudo foi aplicada a lei de Bradford de forma clássica, provavelmente se a mesma aplicação fosse realizada de uma forma mais a fundo, ajustes seriam necessários para que os resultados chegassem ao ideal da lei de Bradford, e alguns pontos seriam mais bem trabalhados para chegar à perfeição correta da aplicação da lei.

Com relação à aplicação da Lei de Bradford nesse estudo, verificou-se a existência de três zonas que representaram a distribuição das publicações científicas dedicadas a Open Government nos periódicos científicos constantes na base de dados $W o S$ entre 2010 a 2016, podendo ser concluída a dispersão conforme as regras da lei.

Já no gráfico 1, apresentado nos resultados desse estudo, pode-se concluir que a tendência global é que muitos países em pouco tempo já estejam aptos para garantir os benefícios de dados abertos e níveis reais de implementação e impacto das iniciativas desses dados, ou seja, inúmeros setores de atividade já existem para quais os dados abertos já estão proporcionando iniciativas que auxiliam em áreas sociais e econômicas.

Outro ponto importante a ser ressaltado, já que uma métrica da bibliometria foi utilizada nesse estudo, é mencionar a existência das métricas aplicadas para Open Government, mais conhecidos como "cinco estrelas", modelo de avaliação de dados abertos baseado nos conceitos de Linked open data, com o objetivo de qualificar as publicações de dados abertos, criado por Berners-Lee (2009), que cabe mencionar neste estudo em questão. Nesse sentido, pode-se concluir que dados abertos conectados permitem que haja o compartilhamento dos dados auxiliando as iniciativas de publicação de dados governamentais, de tal modo que todos os dados conectados ajudam a incrementar a nuvem de Linked Open Data (Bizer; Heath; Berners-Lee, 2011).

Para finalizar e concluir esse estudo, é importante mencionar que as competências dos dados abertos produzidos pelo governo, geralmente são aceitos como dados coletados no exercício de suas competências institucionais, como atividades que não identifiquem pessoas individualmente ou violem a sensitividade comercial. Dados abertos governamentais é um subconjunto das informações do setor público, o qual é mais amplo em seu escopo. 
Devido aos resultados encontrados, sugere-se para trabalhos futuros, a utilização de outras bases de artigos para o termo open government aqui pesquisado, e a utilização de toda a base para a análise qualitativa. Sugere-se, também, a análise de citações sobre o termo, para maior compreensão da produção científica e aumentar a abrangência do estudo. Outra sugestão válida é o estudo qualitativo das publicações feitas pelos autores em relação ao termo pesquisado que mais publicaram artigos no período estudado, para observar se são novas publicações de fato, ou somente outras partes de um estudo já publicado. Realizar estudos dos Colégios invisíveis relacionados ao termo pesquisado em outros periódicos que apresentam estratos elevados no Qualis da CAPES, seria outra sugestão de um trabalho futuro que contribuiria para sociedade acadêmica.

\section{NOTAS}

${ }^{1}<$ https://www.journals.elsevier.com/government-information-quarterly>.

\section{REFERÊNCIAS}

BERNERS-LEE, T. Linked Data. 2009. Disponível em: <http://www.w3.org/DesignIssues/LinkedData.html>. [Consulta: 8 de janeiro de 2018].

BIZER, C.; HEATH, T. e BERNERS-LEE, T. Linked data-the story so far. Semantic services, interoperability and web applications: emerging concepts. Em: Heath, T., Hepp, M., and BIZER, C. (eds.). Special Issue on Linked Data, International Journal on Semantic Web and Information Systems (IJSWIS), 2011, p. 205-227. Disponível em: <http://linkeddata.org/docs/ijswis-special-issue>. [Consulta: 12 de janeiro de 2018].

BRADFORD, S.C. Sources of Information on scientific subjects. Engineering: an Illustrated Weekly Journal, 1934, vol. $137, \mathrm{n}^{\circ} 3550$, p. $85-86$.

BROOKES, B.C. Bradford's law and the bibliography of science. Nature, 1969, vol. 222 (dec.), p. 953-956.

EGGHE, L. A note on different Bradford multipliers. Journal of the American Society for Information Science, 1990, vol. 41, no 3, p. 204-209.

EGGHE, L.; ROUSSEAU, R. Introduction to Informetrics: Quantitative Methods in Library, Documentation and Information Science. Amsterdam: Elsevier, 1990.

EGGHE, L. The dual of Bradford's law. Journal of the American Society for Information Science, 1986, vol. 37, nº 4, p. 246-255.

FERRER-SAPENA, A.; PESET, F. e ALEIXANDRE BENAVENT, R. Acceso a los datos públicos y su reutilización: open data y open Government. El Profesional de la Información, 2011, vol. 20, nº 3, p. 260-269.

GÜEMES, M.C. e RAMÍREZ-ALUJAS, A.V. Gobierno abierto, reforma del Estado y modernización de la gestión pública: alcances, obstáculos y perspectivas en clave Latinoamericana. Em: Hofmann, A.; Ramírez-Alujas, A. V.; Perez-Nieto, J.A.B. (Org.). La promesa del gobierno abierto. México, DF: Instituto de Acceso a la Información Pública y Protección de Datos Personales del Distrito Federal, 2012.

HELBIG, N. et al. The dynamics of opening government data. Center for Technology in Government, 2012. Disponível em: <https://www.ctg.albany.edu/publications/reports/opendata/opendata.pdf $>$. [Consulta: 10 de fevereiro de 2018].

LEIMKUHLER, F.F. The Bradford distribution. Journal of Documentation, 1967, vol. 23, nº 3, p. 197-207.

LUNA-REYES, L.F. e GIL-GARCIA, R.J. Understanding the Co-Evolution of Institutions, Technology, and Organizations: The Enactment of the State Government Portal of Puebla. Em: Proceedings of the 14th Annual International Conference on Digital Government Research - DGO. Quebec, Canadá, 2013.

OKUBO, Y. Bibliometric Indicators and Analysis of Research Systems: Methods and Examples. OECD Science, Technology and Industry Working Papers, 1997/01, OECD Publishing Systems, 70 p. Disponível em: $<$ http://dx.doi.org/10.1787/208277770603>. [Consulta: 16 de dezembro de 2017].

PELED, A. Re-Designing Open Data 2.0. eJournal of eDemocracy and Open Government, 2013, vol. 5, n 2, p. 189199.

PINHEIRO, L.V.R. Lei de Bradford: uma reformulação conceitual. Ciência da Informação, 1983, vol. 12, nº 2, p. 5980.

PRITCHARD, A. Statistical bibliography or bibliometrics? Journal of Documentation, 1969, vol. 24, n 4, p. $348-349$.

TAGUE-SUTCLIFFE, J. An introduction to informetrics. Information Processing \& Management, 1992, vol. 28, ${ }^{\circ}$ 1, p. 1-3.

YU, H. e ROBINSON, D.G. The new ambiguity of -open government. UCLA Law Review Discourse, 2012, vol. 59, p. 178-208. 